\title{
Communicable Diseases Report, NSW, September and October 2012
}

\author{
Communicable Diseases Branch \\ Health Protection NSW
}

For updated information, including data and facts on specific diseases, visit www.health.nsw.gov.au and click on Public Health and then Infectious Diseases. The communicable diseases site is available at: http://www.health.nsw.gov.au/ publichealth/infectious/index.asp.

Figure 1 and Tables 1 and 2 show notifications of communicable diseases with onset in September and October 2012 in New South Wales (NSW).

\section{Enteric infections \\ Outbreaks of suspected foodborne disease}

Eight outbreaks of gastrointestinal disease, thought to be due to consumption of contaminated food and which affected at least 80 people, were reported in September and October 2012. These outbreaks were linked to restaurants or cafes (4), food supplied by caterers (2) and private residences (2). Salmonella Typhimurium (STm) was found to be the cause for three outbreaks; the implicated food vehicles in two of these outbreaks were raw egg mayonnaise and chicken. In one outbreak multiple foods were consumed and the vehicle was unable to be determined. In another outbreak affecting six groups, norovirus was identified as the cause and oysters were identified as the food vehicle. In other outbreaks the causative organism could not be identified due to either lack of sampling of ill people or of suspected food vehicles.

The Salmonella outbreaks were identified by monitoring of clusters of STm through molecular typing (multiplelocus variable tandem repeat analysis - MLVA). A cluster of 45 cases of a STm MLVA subtype was investigated in September and October. Thirty-five cases of STm were interviewed; $88 \%$ had consumed pre-cut chicken pieces in the 3 days prior to onset of illness. Cases continue to be notified and the investigation in collaboration with the NSW Food Authority is ongoing.

\section{Viral gastrointestinal disease}

There were 180 outbreaks of gastroenteritis in institutions reported in September and October 2012, affecting at least 2944 people. The previous 5-year average for this period was 148 outbreaks. A total of 87 outbreaks occurred in aged-care facilities, 75 in child-care centres, 15 in hospitals and three in other facilities. In the $83(46 \%)$ outbreaks in which a stool specimen was collected, norovirus was confirmed in cases from $29(16 \%)$ outbreaks and rotavirus was confirmed in 20 (11\%).

\section{Respiratory infections \\ Influenza}

Influenza activity, as measured by the number of people who presented with influenza-like illness to 59 of the state's largest emergency departments, returned to preseasonal levels in September and October after a peak in mid-July. In addition, the number of people who tested positive for influenza A by diagnostic laboratories decreased to pre-seasonal levels throughout September and October, after a peak in late June. The number of people who tested positive for influenza B also decreased over the reporting period. While this number has yet to return to pre-seasonal levels, it is well below the peak reached in June.

In September, there were:

- 196 presentations to emergency departments (rate 1.3 per 1000 presentations)

- 774 cases of laboratory-confirmed influenza including: - 269 (35\%) influenza A

○ $506(65 \%)$ influenza B.

In October, there were:

- 139 presentations to emergency departments (rate 0.7 per 1000 presentations)

- 260 cases of laboratory-confirmed influenza including: - $42(16 \%)$ influenza A - $218(84 \%)$ influenza B.

For a more detailed report on respiratory activity in NSW see: http://www.health.nsw.gov.au/PublicHealth/ Infectious/influenza_reports.asp.

\section{Vaccine-preventable diseases \\ Meningococcal disease}

Eleven cases of meningococcal disease were notified in NSW in September and October 2012 (seven in September and four in October), a decrease from 15 notified in the 
same period in 2011. The age of the cases ranged from 5 months to 87 years and included six cases aged less than 5 years. There were no deaths due to meningococcal disease notified in this period. Seven $(64 \%)$ cases were due to serogroup B (for which there is no vaccine), three $(27 \%)$ were due to serogroup W135, and one (9\%) was unable to be typed. Of the 15 cases notified during the same period in 2011 , nine $(60 \%)$ were due to serogroup B, one (7\%) was due to serogroup $\mathrm{Y}$, one $(7 \%)$ was due to serogroup $\mathrm{C}$ and the remaining four $(27 \%)$ were of an undetermined serogroup.

It is recommended that a single dose of vaccine against meningococcal $\mathrm{C}$ disease be given to all children at the age of 12 months as well as persons at high risk of disease. ${ }^{1}$

\section{Measles}

Seventy-seven cases of measles were notified in NSW in September and October 2012; all were part of an ongoing outbreak that began in April. This was an increase compared to the six cases reported for the same period in 2011. The age groups most affected were children aged $0-4$ years $(n=24)$, of which 13 were aged less than 1 year, and young adults aged $30-34$ years $(n=10)$. The average age of cases was 17.5 years (range: 6 months-61 years), and $49 \%$ were female. Six $(8 \%)$ cases were Aboriginal people.

Most cases were notified in South Western Sydney Local Health District $(71 \%)$, followed by Western Sydney (Parramatta) (17\%), however outbreak-associated cases were also reported in the Illawarra Shoalhaven $(n=4)$, North Coast (Port Macquarie) $(n=3)$ and South Eastern Sydney $(n=1)$ LHDs as well as Justice Health $(n=1)$. Of the 77 notifications, 64 (83\%) were laboratory confirmed.

The majority $(81 \%)$ of the cases were not vaccinated for measles. Of the 15 cases that were reported to have been vaccinated, five were documented as partially or fully vaccinated for age. Of these, one 10-14-year old had received, according to the Australian Childhood Immunisation Register, two doses; two 10-14-year olds had received one dose; and a 9-month old and a 10-month old who were vaccinated as contacts of confirmed cases had received one dose each.

\section{Pertussis}

During September and October, 776 cases of pertussis were notified with onset in the period in NSW, less than one-third of the 2410 cases notified for the same period in 2011. Most cases were in the 5-9-year age group $(n=237)$, followed by the $0-4$-year $(n=140)$ and the 10-14-year age groups $(n=129)$.

Direct protection for young infants remains available through free vaccination for pertussis that is administered at 2, 4 and 6 months of age. The first dose can be provided as early as 6 weeks of age. There is also a booster dose at $3 \frac{1}{2}$ to 4 years. New parents and grandparents should also discuss the benefits of pertussis vaccination for themselves with their general practitioner. ${ }^{1}$

\section{Sexually transmissible infections and bloodborne viruses Gonorrhoea}

The number of gonorrhoea notifications has continued to rise, with a total of 732 laboratory-confirmed cases notified in NSW in September and October 2012; this is an increase of $41 \%$ compared with the same period in $2011(n=519)$. Since January 2012, there has been an average of 312 notifications of gonorrhoea per month, with a peak of 361 notifications in May.

\section{Syphilis}

A total of 95 cases of syphilis were notified to NSW Health with onset in September and October 2012, fewer than the 148 cases notified in the same period in 2011. A reporting delay may account for some of this decrease.

\section{Lymphogranuloma venereum}

NSW Health was notified of 12 cases of lymphogranuloma venereum with onset in September and October 2012, an increase from the six cases reported in the same period in 2011.

\section{Reference}

1. National Health and Medical Research Council. The Australian Immunisation Handbook. 9th ed. Canberra: Australian Government Department of Health and Ageing; 2008. 
Figure 1. Reports of selected communicable diseases, NSW, Jan 2004 to Oct 2012, by month of onset.

Preliminary data: case counts in recent months may increase because of reporting delays.

Laboratory-confirmed cases only, except for measles, meningococcal disease and pertussis.

$\mathrm{BFV}=$ Barmah Forest virus infections, $\mathrm{RRV}=$ Ross River virus infections, lab conf $=$ laboratory

confirmed, Men Gp $C$ and $G p B=$ meningococcal disease due to serogroup $C$ and

serogroup $B$ infection, other/unk = other or unknown serogroups.

NB: Multiple series in graphs are stacked, except gastroenteritis outbreaks.

NB: Outbreaks are more likely to be reported by nursing homes and hospitals than by other institutions.

\begin{tabular}{|lr|}
\hline \multicolumn{2}{|l|}{ NSW Population } \\
Male & $50 \%$ \\
$<5 \mathrm{y}$ & $7 \%$ \\
$5-24 \mathrm{y}$ & $27 \%$ \\
$25-64 \mathrm{y}$ & $53 \%$ \\
$65+\mathrm{y}$ & $13 \%$ \\
Rural & $46 \%$ \\
\hline
\end{tabular}

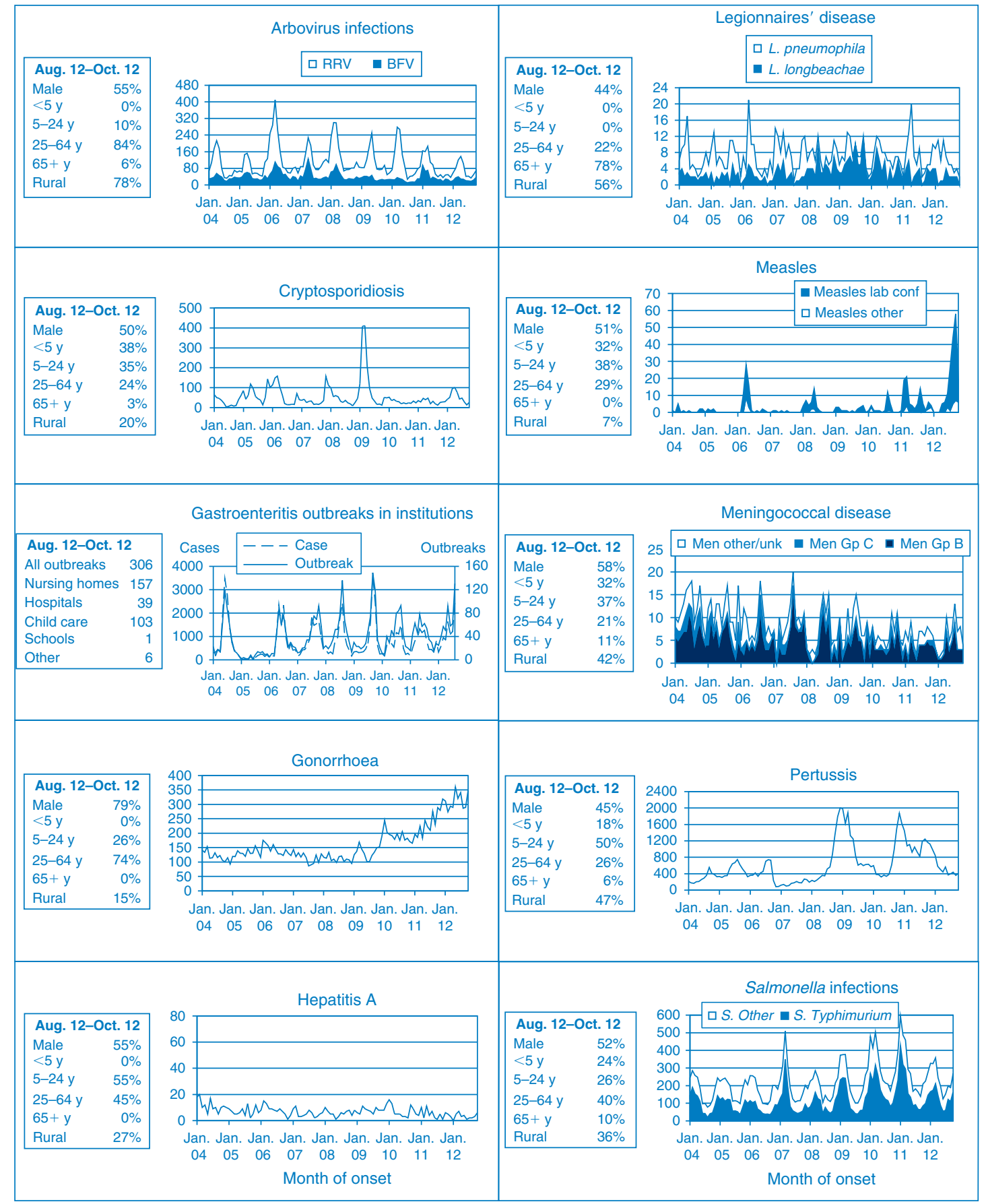




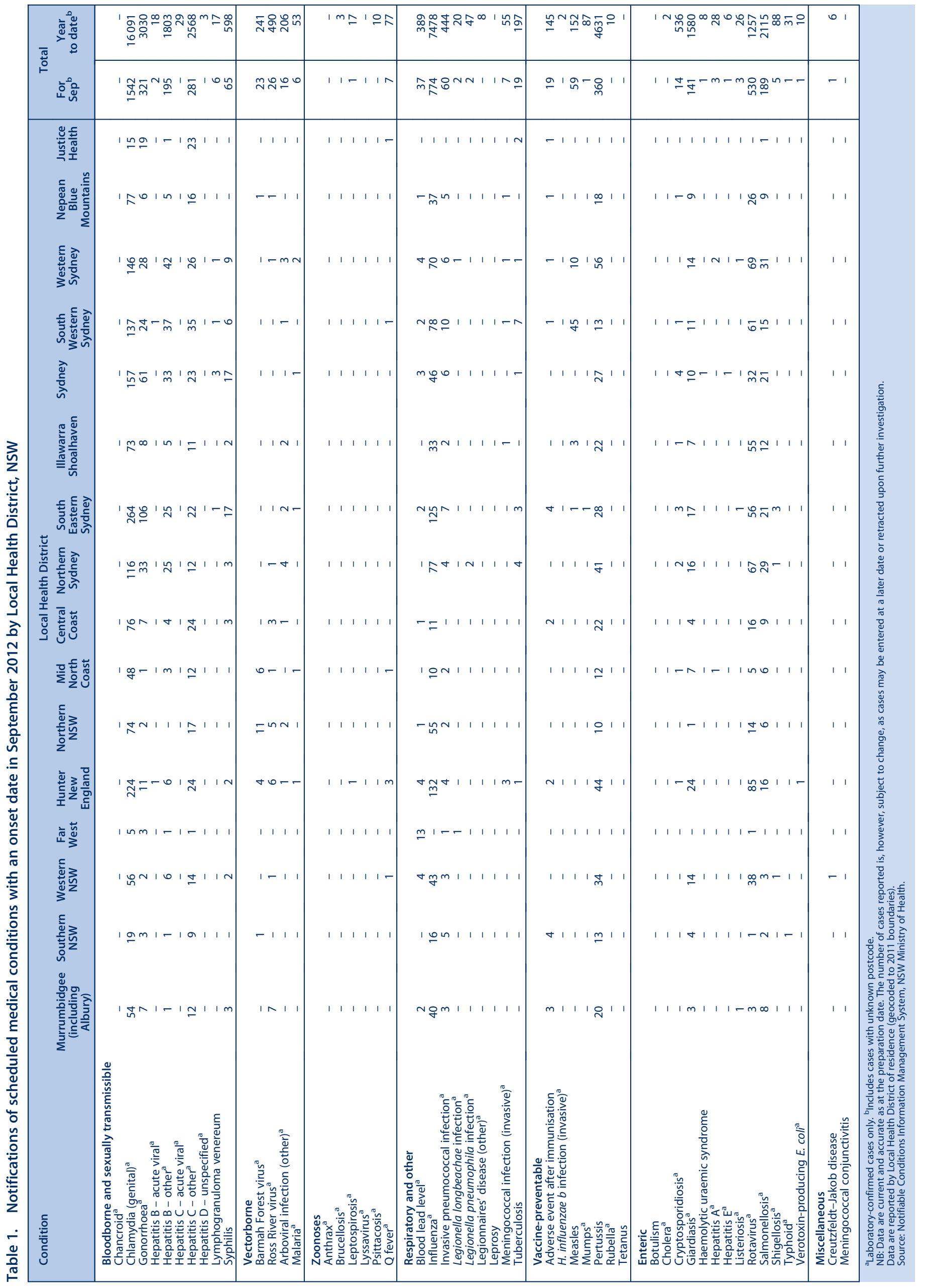

242 | Vol. 23(11-12) 2012 NSW Public Health Bulletin 


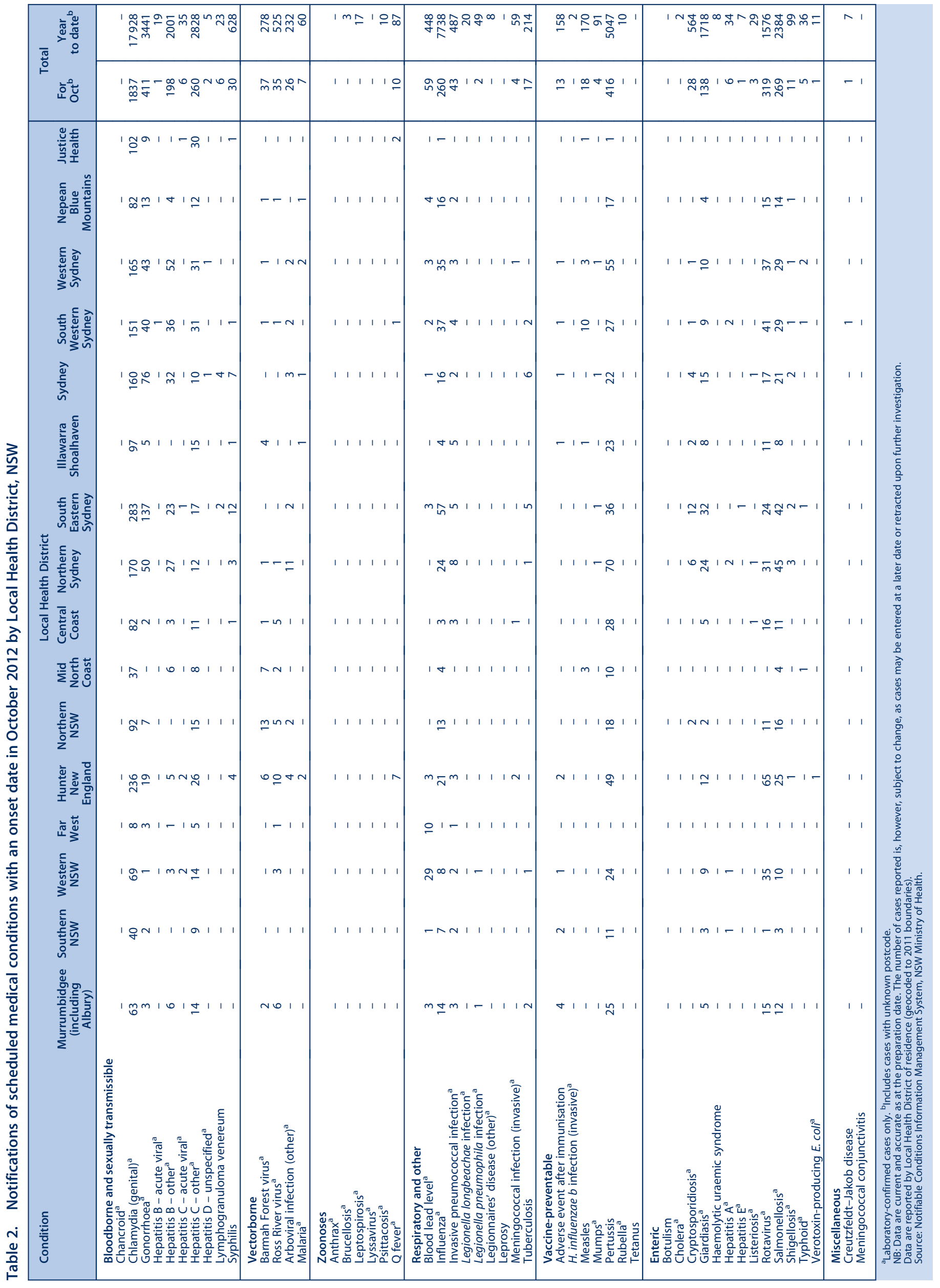

